\title{
A Long term study for upper limb arterio-venous fistula creation for hemodialysis at a tertiary level hospital in Eastern India
}

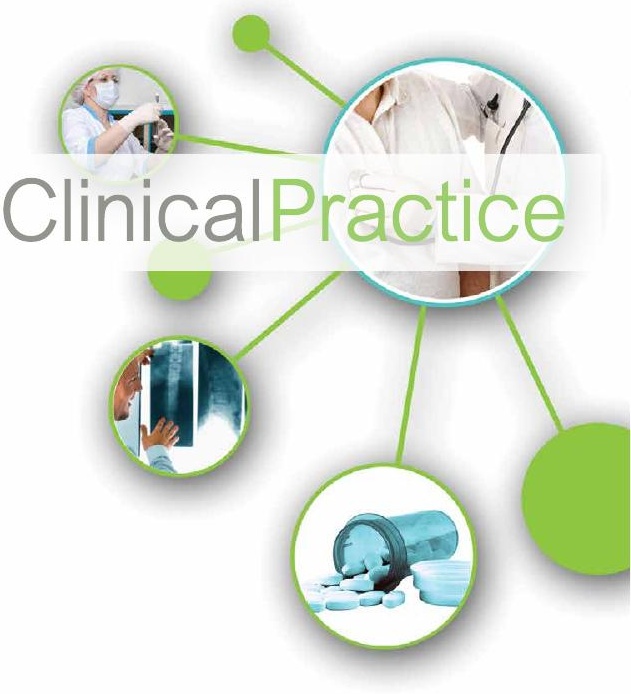

\begin{abstract}
Background: There is gradual increase in need for hemodialysis, as there is gradual increase in the end stage renal disease in India. Permanent vascular access in the patient with end stage renal disease on hemodialysis is provided through a central venous catheter, arteriovenous graft, or arteriovenous fistula. The aim of this study was to evaluate the site, results and postoperative complications of arteriovenous fistula creation in our hospital.

Method: It was a retrograde study conducted at Seth Sukhlal Karnani Memorial (SSKM) hospital, Institute of Post-Graduate Medical Education and Research (IPGMER), Kolkata, between 1st July 2006 and 30th August 2011. All patients, with end stage renal disease requiring long term vascular access for haemodialysis, were included in the study. In most of the patients radio-cephalic fistulae were created in the left forearm, in some on right forearm, just above the wrist joint, and in some patients brachiocephalic or brachiobasilic arteriovenous fistulae were created.

Results: 375 patients were studied; $292(77.86 \%)$ males and 83(22.14\%) females, with male to female ratio of 3.52:1. Distribution of co-morbid factors showed diabetes in 225 (60.0\%), hypertension in 150 (40\%) patients. Radio-cephalic fistula was done in 295 patients and brachiocephalic fistula in 80 patients. In 5\% dominant hand was used and for remaining $95 \%$ of the patients fistula was created on non-dominant hand. Arterio-venous fistula was successful at 6 weeks in $95 \%$, at 2 years in $90 \%$ and at 5 years in $85 \%$ patients. In $5 \%$ cases, failure in first 6 weeks was because of primary failure or wound infections and at 2-5 years failure due to stenosis of cephalic vein by repeated punctures and thrombosis. In failed patients re-do procedure was carried out successfully at another (cubital) site.

Conclusion: Radio-cephalic arteriovenous fistula in patients with end stage renal disease requiring long term vascular access for haemodialysis remains the procedure of choice if done by experienced hands.
\end{abstract}

\section{KEYWORDS: Arteriovenous fistula, radio-cephalic fistula, end stage renal disease, haemodialysis}

\section{Introduction}

The incidence of renal failure is gradually increasing in India. These patients need renal replacement therapy. Renal replacement therapy is provided in the form of dialysis and renal transplant. As the survival after renal failure is increasing because of the availability of renal replacement therapy, there is increased demand for angio access. For temporary angio access central double lumen catheter is passed either into an internal jugular, subclavian or common femoral vein. The most frequent complications of central venous catheter include infection and thrombosis; therefore this access is not recommended. Arteriovenous fistula can be made either at the anatomical snuffbox or the distal, mid or proximal forearm. Sometimes polytetrafluorethylene grafts are placed between the artery and the vein because of the non-availability of good length veins in the superficial tissue. Arterio venous grafts have a high rate of thrombosis and infection. The fistula provides the best outcome. An arteriovenous fistula is created by a surgical anastomosis between and artery and vein. When a fistula is created the vein and artery may be in their normal positions, or the distal end of the vein is moved to a position that is better located for cannulation (vein transposition). A translocation is done when the entire vein is moved from one anatomic location to another requiring an arterial and venous anastomosis. The fistula with the best outcome is the lower arm Radiocephalic Fistula. However this access often fails to mature in the elderly patient with underlying vascular disease, particularly in diabetics [1]. The second recommended fistula is the upper arm brachiocephalic fistula [2]. This type of fistula is being placed with increased frequency because of the high failure rate of Radio cephalic Fistula. The third recommended fistula is the Brachiobasilic fistula [3]. The Radio
Shahbaaz Khan $\mathrm{Md}^{1 *}$ \& Prokash $\mathrm{S}^{2}$

${ }^{1}$ Department of Cardiac surgery, King Fahad Medical City Riyadh, Kingdom of Saudi Arabia

${ }^{2}$ Asst. Prof, Department of Cardiothoracic and vascular surgery, SSKM Hospital, Institute of Postgraduate Medical Education and Research, Kolkata, India

*Author for correspondence:

Tel.: + 966112889999

mohdshahbaazkhan@yahoo.com 
cephalic Fistula was the first fistula designed in 1966 by Brescia [4]. The most frequent clinical problem is that this access has a higher primary failure rate when compared to brachiocephalic fistula or Brachiobasilic fistula However, if a Radio cephalic Fistula matures, the 5 to 10 year cumulative patency rate is 53 and 45 percent respectively [1]. Placement of a lower arm fistula is desirable as it preserves the upper arm for future use. The major complication with a brachiocephalic fistula is the steal syndrome as compared to Radiocephalic Fistula or Brachiobasilic fistula. Because the basilic vein is less accessible to venipuncture it tends to be better preserved and less involved with traumatic post-phlebitic changes when compared to the cephalic vein. Overall, the failure rate of the Brachiobasilic fistula is worse than brachiocephalic fistula or Radiocephalic Fistula. The aim of this study was to evaluate the results arteriovenous fistula in patients of dialysis dependant end stage renal failure in our hospital.

\section{Material and Methods}

This retrograde study was carried out at SSKM hospital, IPGMER Kolkata, over a period of 5 years from 1st July 2006 to 30th august 2011. Total 375 patients were studied. All the patients with end stage renal disease requiring arteriovenous fistula for the first time were included in the study. Data of the individual patients was collected. Age, sex, arterio venous fistula site, co-morbidity, operation time, complications and success of the procedure at 6 weeks, 2 years and 5 years were the parameters considered in this study. The procedure was performed under local anesthesia (xylocaine) with aseptic technique. The wrist site of the left (non-dominant) forearm of the patient was used to create a radiocephalic (brescia-cimino fistula). In our study, the end to side anastomosis technique was used for Radiocephalic arteriovenous fistula but in other types of arterio venous fistula side to side tecknique was used. Prolene 7-0 suture was used for the vascular anastomosis. The arteriotomy and venotomy sites must be carefully chosen and aligned to guard against twisting or distortion when the anastomosis is completed. The anastomosis length is approximately 5 to $8 \mathrm{~mm}$. Suture technique during the anastomosis is very important, particularly at the ends. It should take only small amounts $(1 \mathrm{~mm})$ of tissue during suturing and be very careful at the corners. When an anastomosis is constructed in smaller vessels, the amount of tissue incorporated in the suture becomes exponentially important for successful outcomes [5]. A "back wall first" technique is generally used in these small vessels, similar to the method described by Tellis et al [6]. This involves initiating the suture in the mid portion of the back wall and, using a running suture technique, closing the back wall from the inside of the anastomosis and then completing each corner. The front wall closure is completed with this same continuous running technique, tightening the suture except for the last 2 suture passes, which are placed but left loose to allow air and debris to come out. For the creation of a radiocephalic fistula, a 3-cm longitudinal incision through the skin was made halfway between the cephalic vein and the radial artery at the wrist. The cephalic vein was dissected first, and its collateral branches were then carefully ligated near the main trunk. The dissection was conducted up to the bifurcation of the vein at the dorsal aspect of hand. At this site, the vein was ligated and sectioned. Adequate mobilization is of the utmost importance to prevent kinking after the establishment of the fistula. The adventitia was dissected from the exposed vein to allow for dilatation of the vein by the increased arterial pressure. A thin catheter was introduced into the proximal segment of the vein, and 10 to $20 \mathrm{~mL}$ of heparin saline solution was injected to dilate the vein. The proximal end of the vein was spatulated by performing a 5 to $7 \mathrm{~mm}$ incision in its posterior wall. A $3-\mathrm{cm}$ segment of the radial artery was exposed so that proximal and distal vascular bands could be applied, transecting and ligating all its small branches. The arteriotomy was performed on the anterior wall of the artery utilizing a microsurgical scalpel, and care was taken not to damage the posterior wall of the artery. The incision was completed with microsurgical scissors. A thin catheter was introduced proximally and distally for injection of a heparin saline solution. Two cardinal stitches were made in the proximal and distal corner of the arteriotomy, and a side to end arteriovenous anastomosis was performed by using $7 / 0$ prolene running sutures. After the release of the vascular bands, pulsation and thrill were immediately palpated at the site of the fistula. The skin incision was closed with nonabsorbable $3 / 0$ separate sutures. In the patients in whom the cephalic vein at the forearm was not available, or in whom a previous radiocephalic fistula had failed, the second option was to create a fistula at the elbow. A $3-\mathrm{cm}$ transverse incision across the antecubital fossa was made, 
and the basilica or cephalic vein was exposed, with special care to avoid damage to the medial cutaneous nerve of the arm and side to side anastomosis performed.

\section{Results}

375 patients were studied. All patients were operated under local anesthesia. 270 (72 \%) patients were of age $<50$ years, while $105(28.0 \%)$ were aged $>50$ years. $292(77.86 \%)$ males and $83(22.14 \%)$ females, with male to female ratio of 3.52:1. The operation time ranged from 45 to 90 minutes. The complication rate was very low. $225(60.0 \%)$ patients were diabetic. 150 (40\%) patients were hypertensive. Radiocephalic fistula was created in the left forearm just above the wrist joint in 295 patients and Brachiocephalic fistula in 80 patients. In 5\% dominant hand was used and for remaining 95\% patients, fistula was created on non-dominant hand. Arterio venous fistula was successful at 6 weeks in $95 \%$, at 2 years in $90 \%$ and at 5 years in $85 \%$ patients. In $5 \%$ cases, failure in first 6 weeks was because of primary failure or wound infections and at 2-5 years failure due to stenosis of cephalic vein by repeated punctures and thrombosis. In failed patients re-do procedure was carried out successfully at another (cubital) site.

\section{Discussion}

There are two major considerations in selecting the type of vascular access. First, the new access must mature adequately to be suitable for dialysis. Second, once maturation has been achieved, the access should maintain long-term patency with a minimum of percutaneous or surgical interventions [7]. Side to side fistula is clearly shown to be at greater risk of complications. With the side to side cimino fistula a higher incidence of both arterial steal syndrome and venous hypertension is reported $[8,9]$. Cardiac failure and arterial steal syndrome are commonly described complications with brachial artery fistulae. Early failure of arterio venous fistula may result from non-maturation or thrombosis. Early failure was reported to be $40 \%[10]^{(10)}-55 \%$ [11] in the American series and about $7 \%$ [12] ${ }^{(12)}$ to $10 \%[13]$ in the European series. In our series it was $5 \%$. However, once the fistula is established and functioning well, it rarely fails [14].

\section{Physiology of a fistula access}

The creation of a fistula results in blood flow from an artery to a vein that is inherently non physiologic in many ways. The initial flow rate in the radial artery of $20-30 \mathrm{ml} / \mathrm{min}$ increases to $200-300 \mathrm{ml} / \mathrm{min}$ immediately after creation of an arterio-venous fistula, reaching flow rates of $600-1200 \mathrm{ml} / \mathrm{min}$ after maturation [9]. In addition, the blood flow in the vein is not pulsatile prior to fistula insertion, whereas it is after the fistula is created. High fistula blood flow, a prerequisite for venous dilation and a requirement for easy cannulation and adequate dialysis, is accompanied by high arterial pressure being transmitted to the vein. This intense increase in flow rate and pressure has a profound effect on the hemodynamics in the downstream vein The dramatically increased arterial blood flow at the time of fistula creation ultimately leads to an overall increase in shear stress; early on an observed low shear stress is evident which is thought to contribute to intimal hyperplasia and resultant venous stenosis and ultimate thrombosis.

\section{Complications}

\section{- Early failure}

Early failure of an arterio venous fistula is defined as a fistula which never matures or is unable to be used by three months of time. Causes of early fistula failure are inadequate arterial supply, anastamotic stenosis or outflow obstruction of the venous segment. Other factors contributing to the primary failure include demographic factors such as age, obesity, female sex, diabetes or peripheral vascular disease [15]. The size of the underlying vein may also influence the ability of a fistula to mature. A cephalic vein diameter of less than 2.0 $\mathrm{mm}$ in the forearm and less venous distensibility increases the risk of primary failure [16]. Cause of poor maturation is the development of collateral circulation. The Dialysis outcomes practice patterns study (DOPPS) noted a lower risk of failure of established fistulas in patients who used aspirin consistently over a year [17]. The Dialysis access consortium fistula trial (DAC) showed that clopridrogel reduced the risk of fistula thrombosis by $37 \%$ [18]. In the DAC study $61 \%$ of newly created fistulas failed. These findings and others have shown a primary failure rate of $31-61 \%$ [18-20].

\section{- Late failure}

Late failure of the fistula is defined as failure occurring greater than three months after creation and is often due to outflow stenosis. Venous stenosis is usually detected clinically by 
symptoms of swelling of the extremity, prolonged bleeding post dialysis, and difficult cannulation. The most common anatomic location for an outflow stenosis in a Radiocephalic Fistula is 3 $\mathrm{cm}$ from the arteriovenous anastomaosis [21]. Outflow stenosis in Radiocephalic Fistula may be treated successfully by angioplasty with favorable primary and secondary patency rates [25]. Inadequate arterial flow is often detected. An arterial lesion may be present in $15-30 \%$ of fistula [22]. This type of lesion also is successfully treated by angioplasty or surgical revision [23]. One of the causes of failure of brachiocephalic fistula is due to stenosis in the cephalic arch, which is the final bend in the cephalic vein prior to entry into the axillary vein. Cephalic arch stenosis (CAS) is found to occur in up to $77 \%$ of patients with brachiocephalic fistula compared to $30 \%$ with Radiocephalic Fistula. The risk of development of Cephalic arch stenosis is less in diabetics for unclear reasons [24]. The Brachiocephalic fistula has been shown to be a superior access in older diabetic patients [25].

\section{- Aneurysms}

The incidence of aneurysm formation in fistulas varies in studies from 5-7\% [26]. Aneurysms form for many reasons which include repeated cannulation or altered turbulent blood flow from stenosis.

\section{- Steal syndrome}

Steal syndrome is defined as distal hypoperfusion of the extremity in patients with severe peripheral vascular disease due to shunting of arterial blood flow into the fistula [22]. Reverse flow occurs if the diameter of the fistula opening is greater than the diameter of the feeding artery. Symptomatic steal occurs when there is a failure of adequate collateral flow. This problem complicates approximately $3-5 \%$ of fistulas and grafts. It is likely to occur more frequently with Brachiocephalic fistula (6\%) verses Radiocephalic Fistula. Hand

ischemia from steal syndrome may require Distal Revascularization with Interval Ligation procedure or complete ligation in severe cases. The Distal Revascularization with Interval Ligation (DRIL) procedure was first proposed by Harry Schanzer in 1988 [27].

\section{- Infection}

Predisposing factors to infection include: inadequate skin disinfection prior to cannulation, pseudo aneurysms, peri-fistular hematomas, and pruritis with skin excoriation, or the use of the fistula for intravascular drug use.

\section{- Cardiac failure}

Creation of a fistula is associated with a $15 \%$ increase in cardiac output and $4 \%$ increase in left ventricular end-diastolic diameter. High output failure from fistula access occurs in less than $1 \%$ of cases.

\section{Median nerve injury}

It may occur from ischemic injury from steal, compression of the nerve if there is extravasation of blood or local amyloid deposition in long term dialysis patients. If traditional therapy to treat neuropathy does not resolve the pain, the fistula may need to be ligated.

\section{Conclusion}

Radio-cephalic Arterio-venous fistula in patients with end stage renal disease requiring long term vascular access for haemodialysis remains the procedure of choice if made by experienced surgeons. Mechanical dilatation of the artery and vein, before starting the anastomosis, as well as the use of vasodilatory agents, could decrease early thrombosis of the fistula, and this method has very high early patency. If a patient starts hemodialysis with a mature fistula, their transition to renal replacement therapy occurs with less risk of morbidity and mortality.

4. Brescia Mj, Cimino Je, Appel K et al. Chronic haemodialysis using venepuncture and a surgically created arteriovenous fistula. $N$. eng. j. med. 275, 1089-92 (1966)

5. Jennings WC, Wood CD. Role of vein patch angioplasty in isolated operations for profunda femoris stenosis and disabling claudication. Am. J. Surg. 150, 263-265 (1985).

6. Tellis VA, Veith FJ, Sobermann RJ et al. Internal arteriovenous fistula for hemodialysis. Surg. Gynecol. Obstet. 132: 866-870 (1971).

7. Allon M, Robbin Ml. Increasing arteriovenous fistulas in hemodialysis patients: problems and solutions. Kidney. int. 62, 1109-24 (2002).

8. Haimov M. Vascular access for hemodialysis. Surg. Gynaecol. Obstet. 141, 619-25 (1975).

9. Wedgwood Kr, Wiggins Pa, Guillou PJ. A prospective study of end to side vs. Side to side arteriovenous fistulae for haemodialysis. Br. J. surg. 71, 640-2 (1984).

10. Tordior JHM, Rooyens P, Dammers R et al. Prospective evaluation of failure modes in autogenous radiocephalic wrist access for haemodialysis. Nephrol. Dial. Transplant. 18, 378-383 (2003). 
11. Miller PA, Tolwani A, Luscy P et al. Predictor of adequacy of arteriovenous fistulas in hemodialysis patients. Kidney. Int. 56, 275-280 (1999).

12. Koo SL, Burnapp L. Contemporary vascular access surgery for chronic haemodialysis. J. $R$. Coll. Surg. Edinb. 41, 164-169 (1996).

13. Reilly DT, Wood RF, Bell PR. Arteriovenous fistulas for dialysis: blood flow, viscosity, and long-term potency. World. J. Surg. 6, 628-633 (1982).

14. Palder ST, Kirkman RL, Whittemore AD et al. Vascular access for hemodialysis. Ann. Surg. 202, 235-239 (1986).

15. Huijbrets HJ, Bots ML, Wittens Ch et al. Hemodialysis arteriovenous fistula patency revisited: results of a prospective, multicenter initiative. Clin. J. Am. Soc. Nephrol. 3(3), 714721 (2008).

16. Silva MB, Hobson RW, Pappas PJ et al. A strategy for increasing use of autogenous hemodialysis access procedures: impact of preoperative noninvasive evaluation. J. Vasc. Surg. 27(2), 302 (1998).

17. Hasegawa T, Elder SJ, Bragg-Gresham JL et al.
Consistent aspirin use associated with improved arteriovenous fiatula survival among incident hemodialysis patients in the dialysis outcomes and practice patterns study. Clin. J. Am. Soc. Nephrol. 3(5), 1373-8 (2008).

18. Dember LM, Beck GJ, Alton $M$ et al. Effect of clopidrogigrel on early failure of arteriovenous fistulas for hemodialysis: a randomized controlled trial. JAMA. 299(18), 2164-2171 (2008).

19. Biuckians A, Scott EC, Meier GH et al. The natural historyof autologous fistulas as firsttime dialysis access in the K-DOQI era. J. Vasc. Surg. 47(2), 415-421 (2008).

20. Schilid AF, Prieto J, Glenn M et al. Maturation and fistula rates in a large series of arteriovenous dialysis access fistulas. Vasc. Endovasc. Surg. 38(5), 449-453 (2004).

21. Rajan DJ, Bunston S, Misra S et al. Dysfunctional autogenous hemodialysis fistulas: outcomes after angioplasty-are there clinical predictors of patency? Radiology. 232(2), 508515 (2004).

22. Leon C, Asif A. Arteriovenous access and hand pain: the distal hypoperfusion ischemic syndrome. Clin. J. Am. Soc. Nephrol. 2(1), 175183 (2007).

23. Turmel-Rodrigues L, Pengloan J, Rodrigue $\mathrm{H}$ et al. Treatment of failed native arteriovenousfistulae for hemodialysis by interventional radiology. Kidney. Int. 57(3), 1124-1140 (2000)

24. Hammes M, Funaki B, Coe FL. Cephalic arch stenosis in patients with fistula access for hemodialysis: relationship to diabetes and thrombosis. Hemodialysis Int. 12(1), 85-89 (2008).

25. Papanikolaou V, Papagiannis A, Vrochides D et al. The natural history of vascular access for hemodialysis: A single center study of 2,422 patients. Surgery. 145(3), 272-9 (2009).

26. Lo HY, Tan SG. Ateriovenous fistula aneurysmplicate, not ligate. Ann. Acad. Med. Singapore. 36(10), 851-853 (2007).

27. Schanzer H, Schwartz M, Harrington E et al. Treatment of ischemia due to "steal" by arteriovenous fistula with distal artery ligation and revascularization". J. Vasc. Surg. 7(6), 770773 (1988). 